\title{
Validation of Computational Analysis Procedures with Respect to Product Liability and Consequential Loss*
}

\author{
by Anup Puri**
}

\section{Summary}

Many organisations use validation procedures to a certain degree as a means of controlling accuracy and standards in their products and operations to meet Product Liability laws.

The paper presents a role model for a validation system designed to fulfil such requirements. Validation aspects of the following topics are covered; management of analysis operation; acquisition, development and verification of software; qualification and documentation of analysis methods; education and training of personnel.

\section{Introduction}

This paper states the principles that can be applied in the validation of computer models (mathematical) and software used in the design of products. The objective is to ensure that such models are validated to a degree appropriate to their intended use and are applied in a consistent and controlled manner for analysis affecting its integrity.

These requirements apply to any analysis type, which contributes to establishing the integrity of Grade A design, or to any analysis which is to be supplied as a deliverable in its own right where it may in future be so used.

\section{Fitness for purpose}

It is not a requirement that every computational analysis has to be performed to the highest degree of accuracy. The key to effective computational analysis is to match the degree of control to the purpose of the analysis. To this end we can define three categories of importance of analyses as either VITAL, IMPORTANT or ADVISORY. These relate to the consequences of failure of the product and the role which the analysis fulfils in the demonstration of its integrity.

\footnotetext{
* Paper presented at the MORE 9 Meeting in Basle, November 1994.
}

** GEC Marconi Defence, UK. 


\section{Overview}

The total computational analysis system is modelled in terms of three serial activities:

- Acquisition, development and verification of analysis and associated software.

- Development and qualification of analysis methods; product design development.

- The management functions which implement and co-ordinate the activities; education, training and experience of personnel.

\section{Acquisition of software}

Software may be developed in-house, developed by a third party to the Design Authority's requirements (subcontracted) or purchased as commercial off-the-shelf packages.

The Design Authority should ensure that software used in the computational analysis conforms with the application analysis requirements, particularly in respect to the analysis type. In acquiring software the Design Authority should define, where possible, the technical requirements and the tests or conditions which demonstrate satisfaction of those requirements.

The validation issues relating to purchased software are:

- Evaluation of the suitability of the software to the analysis requirements of the product and the inherent limitations of the software.

- Verification of the software against its functional specification.

- The software suppliers Software Quality Control system.

- The software technical support service.

\subsection{Evaluation of software}

Software evaluation is part of the product design organisation's quality system in software acquisition. The two sources of preliminary evaluation are the software theory and validation documents. Examination of the theoretical basis and numerical algorithms together with a range of bench mark problems may be sufficient to demonstrate the applicability and limitations of analysis software. Evaluation provides the technical management with the required appreciation of the software limitations.

\subsection{Verification of software}

Software verification is primarily a software suppliers function and should be part of the suppliers Software Quality Control system; however, it is the responsibility of the user to ensure that the verification has been performed. The verification tests must include benchmarks tests (4.1). Basically these demonstrate that the software has satisfied fundamental tests for soundness and convergence - example, element tests for invariance, rigid body modes etc. The tests are examined for complete coverage of the theoretical basis, facilities and numerical algorithms.

\subsection{Suppliers Software Quality Control System}

The most important aspects of the software Suppliers Quality Control System (SQC) relevant to the product user are:

- Control of the software development process. 
- Verification of the software.

- The procedures for identifying, controlling and correcting software errors.

Control of the software development insures against deterioration of the software under continual enhancement. The software quality control system must define the supplier's procedures for detecting, reporting, controlling and rectifying errors.

\subsection{Software support}

The requirements for support of software used in integrity demonstration analyses include:

- Error and correction notification.

- Documentation.

- Training specific to the software.

The software supplier must provide training in the use of the software. This should not merely cover the mechanics of input, but should include such topics as the solution methods used, limitations of the theory, element formulations and algorithms, diagnostic and error messages and their meaning, outline of the programme structure and operation.

\subsection{Internal software}

All internally developed software used in qualified analysis procedures is subject to the same requirements as external software. Use of uncontrolled software is identified as a significant risk.

\subsection{Software identification and traceability}

The release, issue or version of software used to produce each analysis should be identified and recorded. Changes resulting in a new release issue or version should be recorded so as to produce traceability of software evolvement and of software used for different analyses.

\section{Analysis procedure development}

The development of qualified analysis procedures starts with a systematic review of methods in common and established use. Documentation and qualification of those methods form the basis of the analysis procedures library.

\subsection{Documentation of analysis procedures}

The documentation of an analysis procedure includes the following items:

- The output data for which it has been qualified and the order of its accuracy. This is limited solely to the data correlated in the qualification analysis. The order of accuracy is based on the degree of that correlation.

- The scope and limitations of its applicability, defined in sufficient detail to ensure that the procedure is not used outside that scope. Typically these include the identifying characteristics of the physical model, analysis type, limitations of the behavioural modelling and theory assumptions.

- Reference to documented analysis used in qualification of the procedure. 
- The maximum grade of importance of analysis in which the procedure may be used. This is based on the degree and number of reference validation analysis. The grade of importance defaults to "advisory" if the procedure is not qualified.

- The input data required for satisfactory execution of the procedure.

- The software and facilities to be used, e.g. mathematically modelling, solution technique etc.

- The analysis controlled procedures, the level of detail must be sufficient to enable an analysis, of the relevant level of competence, to execute the analysis in a satisfactory manner.

- The QA checks to be exercised within the procedure, pre-analysis checks, analysis execution checks, post-analysis checks.

- Identification of the "procedure owner" responsible for action on errors, omissions and queries.

\subsection{Qualification of analysis procedures}

Analysis procedure qualification is performed by analyses of realistic engineering models for which results, appropriate to the purpose of the analysis, can be confirmed by some independent means. Confirmatory results are typically obtained from physical tests supporting integrity demonstration of previous products, service experience, failure investigations, alternative analyses for third party assessments. Many test results can be found in open literature. The qualification tests are executed to the defined procedure, preferably by an independent analysis of the appropriate level of competence, to ensure that the procedure documentation is adequate. The documentation of a qualification analysis is sufficient to enable it to be repeated without reference to any other documentation so that, if the procedure is amended, the qualification can be repeated with a minimum of delay. Qualification analysis reports are subject to formal issue and change control. The software execution decks used in the qualification analysis are added to the test library for software acceptance.

In assembling the qualification in respect to the grade of importance, both the degree of correlation and the reliability of the confirmatory values are taken into account. The more qualification tests and correlations available, the greater the confidence in the procedure and the higher the grade of importance of analysis in which the procedure may be used. Qualification of analysis procedures provides project management with an appreciation of the inherent assumptions and limitation of the analysis method.

\section{Product analysis}

The product analysis is controlled by the project analysis manager through the project analysis plan. The individual analysis tasks are allocated through analysis specifications which use the analysis procedures qualified in the methods development activity. After checking of the results each analysis is documented, approved by the analysis project manager and used to update or amend the plan.

Details of individual items are given in the following sections. 


\subsection{Project analysis plan}

The project analysis is a dynamic document which starts as a broad outline and becomes more detailed as the design evolves. It is updated and amended as analysis results are obtained and may involve tasks in the methods development activity. At completion of the project the analysis plan becomes a record which correlates the individual analysis reports. The analysis plan is a controlled document subjected to periodic reissue and change control procedures.

Particular QA features of the plan are:

- The identification of decisions or reviews, based on structural features and analysis results, which may result in updating the plan and redirection of analysis activities.

- Quantitative assessments, estimates, design reviews and correlations between analyses to be used in checking the results from individual analysis.

- The scope and grade of importance of each analysis.

Based on the scope of the analysis and its category of importance, each analysis task is allocated to an analysis team (supervisor, analyst and software consultant) who collectively fulfil the requirements of experience and expertise.

\subsection{Analysis specifications}

The analysis team first prepares an analysis specification which is agreed by the project manager before the analysis proceeds. The purpose of the specification is to ensure that the analysis is sufficient to fulfil its purpose, that the appropriate input data is available and that the results are relevant to the project needs. The specification includes:

- The purpose of the analysis and output data required.

- The sources of authentic data for input.

- The qualified analysis procedure to be used.

- The input and results checking procedures to be invoked.

Where a qualified procedure is not available, the procedure itself is outlined in the specification and the results used only for advisory purposes.

\subsection{Results checking}

At completion of the analysis the results are checked and assessed. This involves:

- Confirming that all the analysis procedure quality assurance checks have been executed satisfactorily.

- Comparison of the results with the estimates and correlations identified in the analysis plan. Quantitative estimates are obtained from traditional simplified analysis.

- Assessment of the results based on knowledge of the physical problem. For analysis of high grade of importance, an independent assessment is performed by a qualified individual who is not a member of the analysis team.

\section{Analysis documentation}

The controlled documentation of the analysis consists of an analysis report, the analysis record and project computer data files.

The analysis report provides only the information relevant to product design and integrity. It includes: 
- The purpose of the analysis.

- An outline of the representation of the physical problem by the analysis model.

- Summary, discussion and accuracy assessment of the principle results.

- Relevance of the results to the engineering problem and design recommendations, and

- References to enable further details to be obtained from the analysis records.

Summaries of analysis reports, including the scope of analysis, grade of importance and identification of analyst, supervisor and software consultant are stored on a database. This is used for subsequent task allocation decisions.

The analysis record includes:

- The analysis specification.

- The key input data, in terms of the physical model and mathematical model representation, and the sources of that data.

- Selected output relevant to the purpose of the analysis.

- A summary of QA checks.

- Location of the input in the project computer files and the version/release number of the software used.

The analysis record, together with the stored input files, should be sufficient to enable the analysis to be repeated or updated reliably with a minimum of effort.

The project computer files contain the program input, and where appropriate, output relevant to the analysis. Where a number of design iterations are involved, only that germane to the definitive version is stored. The data to be retained is defined in the job closedown procedures and is stored in secured files.

\section{Personnel requirements}

The requirements are quantified in terms of:

- Formal academic or professional qualifications.

- Product analysis experience.

- Mathematical modelling and problem solving relevant to the scope of the analysis, and

- Relevant software application experience.

The required degree of training and experience varies with the category of importance of the analysis and must be relevant to the scope of the analysis. Formal training in computational methods and software application contributes to personnel accreditation. Job experience, accumulated in one category, contributes to qualification for tasks of a higher category. Thus an analyst may perform low category tasks, under supervision, until sufficient experience is gained to perform such tasks unsupervised. He may then move to a higher category under supervision, and so on.

In cases where the required software application expertise is not available within the product design organisation, it may be necessary to contract suitable personnel from the software suppliers organisation. Similarly if the organisation is subcontracting analysis of an unfamiliar product, the necessary product expertise may be provided by the contractor. 
8.1. Training should be provided by a combination of formal tuition and on job training. It is effective to use two types of course, one in general computational methods and others in the use of particular specialist software packages. This leads to a better appreciation of the analysis technology and maintains flexibility in the use of different codes.

\subsection{Personnel records}

The personnel information should be extracted from the following sources:

- Professionnal qualifications and years of engineering experience from the company personnel records.

- Training course attendance from the training records.

- The category of importance, scope of analysis, identification of the supervisor, analyst and program consultant, from the analysis report summaries.

This information should be co-ordinated in a data base and used in a variety of ways:

- In task allocation. Given the category of importance, scope of analysis and a list of available personnel, all possible teams which fulfil the requirements are returned. This provides a management aid in task allocation and job scheduling. Note that this does not prevent the project manager from rejecting any team which, based on other considerations, is deemed to be inadequate.

- In the provision of training courses. Given the training course type, returns a list of personnel that require the training in order to progress to higher category of importance tasks. Provides a means of scheduling courses.

- In on job training. Given personnel identification, returns list of analysis scope, category of importance and roles, required to advance to higher category tasks. Provides a means of monitoring the advancement of individuals.

\section{Discussion}

The real quality of any computational analysis is determined by technical considerations. It is important to use experienced technical staff in determining computational procedures and using specialist Quality Assurance consultants in an advisory capacity. to:

The recommended first steps in implementing a computational validation system are

- Constitute a technical body with responsibility and authority for the analysis validation system.

- Agree responsibilities, with the organisation, for each activity required in the system.

- Review current practices against the requirements.

\section{Acknowledgements}

The author acknowledges the valuable contributions made to this subject by members of MOD-JTRC Mathematical Modelling Working group and NAFEM-QA group, both of which the author is a prominent working member. 


\section{REFERENCES}

BABUSKA, I, "Reliability of Computational Processes in Structural Mechanics", 6th World Congress on FEM, October 1990, Banff, Canada.

KACPRZYNSKI, J.J., "Reliability Studies of Finite Element Methods in North America”, 1987, AGARD Report No. 784.

PURI, A., "Capability, Accreditation of Personnel Competence in Organizations carrying out Structural Analysis using Finite Element Methods", 3rd Int. Conf. on Structural Failure Product Liability and Technical Insurance, Vol. 2, Nos. 1/2 P280, 1990.

NAFEMS, "Quality Systems Supplement to ISO 9001 relating to Finite Element Analysis in the Design and Validation of Engineering Products", 1990, Dept. of Trade and Industry, NEL, Glasgow.

ISO 9001 Part. 1, "Quality Systems, Part 1 Specification for Design/Development, Production, Installation and Servicing", 1987, British Standards Institution publication.

PURI, A., "Role of Finite Element Analysis in Structural Qualification”, 6th World Congress on FEM, October 1990, Banff, Canada.

RHEINBOLDT, W.C. and BABUSKA, I., "The Reliability and Optimality of Finite Element Methods", Computer and Structures, Vol. 10, 1979, pp. 87-94.

JACKMAN, R.R. and WHITE, P.S., "Review of Benchmark Problems for Nonlinear Material Behaviour", October 1987, NEL, Glasgow.

CHRISFIELD, M.A., HUNT, G.W. and DUXBURY, P.G., "Benchmark Problems for Geometric Nonlinearity" October 1987, DTI-NEL, Glasgow.

BARLOW, J., "Quality Management in Finite Element Analysis”, Draft paper for AGARD, 1990.

BATHE, K.J., "Some issues for Reliable Finite Element Analysis", Reliability of Methods of Eng. Analysis, 1986, pp. 139-159.

PURI, A., "Finite Element Methods - A Black Box Approach to Structural Clearance", Proceedings of 4th Int. Conf. on Structural Failure and Product Liability, July 1992, pp. 260-67.

ZING, J.W., "Survey of Results of Finite Element Quality Control”, Finite Element News, June 1989.

WILSON, R.I. and LLOYDS, I.J., "Software Quality - Some Legal Issues", Proc. IFIP WG 5.4 Working Conf. on Approving Software Products (ASP-90), September 1990, Elsevier.

SEPAHY, D. and PURI, A., Draft Paper on Mathematical Modelling Analysis Procedure Development, MMWP, MOD 1993. 